CARDIOVASCULAR MEDICINE

\title{
Atrial fibrillation after radiofrequency ablation of atrial flutter: preventive effect of angiotensin converting enzyme inhibitors, angiotensin II receptor blockers, and diuretics
}

\author{
W Anné, R Willems, N Van der Merwe, F Van de Werf, H Ector, H Heidbüchel
}

Heart 2004;90:1025-1030. doi: 10.1136/hrt.2003.023069

See end of article for authors' affiliations

.....................

Correspondence to: Dr H Heidbüchel,

Cardiology-

Electrophysiology,

University Hospital

Gasthuisberg, Herestraat

49, B-3000 Leuven,

Belgium; hein.heidbuchel@

uz.kuleuven.ac.be

Accepted

28 November 2003
Objectives: To determine risk factors for the development of atrial fibrillation (AF) after atrial flutter (AFL) ablation; and to study the relation between AF development and periprocedural drug use.

Methods: AFL ablation was performed in 196 patients. The relation between AF occurrence and clinical, echocardiographic, and procedural factors and periprocedural drug use was analysed retrospectively by a Cox proportional hazard method.

Results: After a median follow up of 2.2 years, 114 patients (58\%) developed at least one AF episode. Factors associated with AF development were the presence of preprocedural AF, a history of cardioversion, and the number of antiarrhythmic drugs used before the procedure. Use of angiotensin converting enzyme (ACE) inhibitors/angiotensin II receptor blockers and diuretics was significantly associated by univariate and multivariate analyses with less development of AF.

Conclusions: A high proportion of patients develop AF after AFL ablation. The incidence of AF is related to pre-ablation AF and its persistence. ACE inhibitors/angiotensin II receptor blockers and diuretics seem to protect against AF.
$\mathrm{R}$ adiofrequency catheter ablation of the isthmus between the tricuspid annulus and the inferior vena cava has become the first line of treatment for recurrent atrial flutter (AFL). ${ }^{1}$ Ablation has evolved into a highly effective and safe procedure. Nevertheless, the immediate and long term success of AFL ablation is often offset by the occurrence of atrial fibrillation (AF). ${ }^{2-10}$ These two arrhythmias are often associated, since they share the same risk factors. Since AF occurs in $12-54 \%$ of patients after successful AFL ablation, ${ }^{2-10}$ one may wonder for which patients AFL ablation is a meaningful intervention. Moreover, better identification of patients who are at risk for the development of AF may help to optimise the antiarrhythmic strategy after AFL ablation.

There is growing evidence, both from animal work and clinical studies, of the involvement of the angiotensin system in the pathophysiology of AF. Angiotensin converting enzyme (ACE) expression is increased in atrial biopsies of patients with $\mathrm{AF}^{11}$ and angiotensin II concentrations are increased in animal models of AF. ${ }^{12}{ }^{13}$ Prior studies have shown that ACE inhibitors diminish the incidence of AF after myocardial infarction and in the setting of heart failure, and that angiotensin II receptor blockers (ARBs) reduce the recurrence of AF after cardioversion for persistent AF. ${ }^{14-16}$ The purpose of this retrospective study was to identify the risk factors for the development of AF after ablation of AFL and to study which drugs reduced AF recurrence during follow up, with emphasis on the role of the renin-angiotensin pathway.

\section{METHODS}

\section{Patients}

We report on 267 consecutive patients who underwent radiofrequency catheter ablation for AFL at the University Hospital Leuven, Belgium, between February 1995 and March 2002. Sixty five patients were excluded from analysis because of concomitant ablation of pulmonary veins or another intra-atrial re-entrant tachycardia during the same procedure. Six patients were excluded because less then one month of follow up was available. One hundred and ninety six patients were eligible for analysis.

The patients were divided into four groups: group l consisted of patients with only AFL before the ablation procedure $(\mathrm{n}=84)$; in group $2 \mathrm{AFL}$ was the dominant presenting arrhythmia but AF was occasionally observed $(\mathrm{n}=49)$; in group $3 \mathrm{AF}$ was the dominant arrhythmia, with intermittent organisation into AFL $(\mathrm{n}=19)$; group 4 consisted of patients with class Ic flutter-that is, conversion of AF into flutter by treatment with class I drugs $(\mathrm{n}=24)$ or amiodarone $(\mathrm{n}=20) \cdot{ }^{17}$

\section{Electrophysiological study and ablation procedure}

Ablation was performed under general anaesthesia with propofol. Antiarrhythmic drug treatment was continued until ablation. Right and left atrial activation were recorded with a duodecapolar catheter (Halo, Cordis Webster, Baldwin Park, California, USA) and multipolar coronary sinus catheter, respectively. AFL was induced by rapid atrial pacing from the proximal coronary sinus. Entrainment pacing confirmed participation of the inferior isthmus in the circuit. Ablation was performed during flutter $(n=22)$ or after restoration of sinus rhythm $(\mathrm{n}=174)$.

Ablation was guided by right atrial angiography. ${ }^{18}$ Acute success was defined by the presence of bidirectional isthmus block persisting until the end of the procedure (that is, at least 30 minutes).

Patients were anticoagulated before ablation based on the same clinical parameters as used in patients with AF. Before the ablation, treatment was converted to low molecular weight heparin. After ablation low molecular weight heparins were restarted until oral anticoagulation again reached an international normalised ratio (INR) $\geqslant 2$. The other patients received aspirin $300 \mathrm{mg} /$ day from the day before to at least six weeks after ablation.

Abbreviations: $A C E$, angiotensin converting enzyme; $A F$, atrial fibrillation; AFL, atrial flutter; ARB, angiotensin II receptor blockers 


\section{Follow up}

Patients received a Holter monitor for the first 24 hours after ablation. The preprocedural antiarrhythmic drug was unchanged until at least the first ambulant visit after six weeks. Further follow up information was obtained from outpatient visits, from reports of referring cardiologists (at least within three months), or by telephone or questionnaire contact with the patient. Since all patients had symptomatic AF before ablation, any symptoms suggestive of AF recurrence were logged as such onto the database, unless otherwise proved (for example, by negative repeat Holter or event recordings during "symptoms" reported by the patient). Apart from six weeks' Holter monitoring, unless there was an obvious recurrence, patients were not routinely monitored. Cessation of antiarrhythmic drugs was considered only if recurrences were excluded by the absence of symptoms and negative Holter results.

\section{Statistical analysis}

Continuous and normally distributed data are given as mean (SD) and non-normally distributed data as median (interquartile range). Comparisons between groups for categorical variables were based on the $\chi^{2}$ test. Comparison between groups for continuous variables were made with one way analysis of variance and in the case of significant difference this was further analysed with the Tukey-Kramer test. Results were considered to be significant at $\mathrm{p}<0.05$.

The actuarial probability of freedom from AF after AFL ablation was calculated with the Kaplan-Meier method and the differences between the curves were tested for significance by log rank statistics. Relative risk calculation and multivariate analysis were done by the Cox proportional hazard method. The identification of the various predictors of post-ablation AFL was based on a stepwise backward selection method. The influence of various drugs was analysed on the basis of an intention to treat. ACE inhibitors, ARBs, or diuretics should have been taken for at least one month; otherwise these patients were excluded from analysis. Statistical analyses were undertaken with a statistical package (StatView version 5.01, SAS institute Inc, Cary, North Carolina, USA).

\section{RESULTS}

\section{Patient characteristics and ablation outcome}

Table 1 summarises baseline patient characteristics. Structural heart disease was present in 51\% (101) of patients. Hypertension (34 patients) and coronary heart disease (30 patients) were most common. The mean left atrial diameter $(4.0(0.6) \mathrm{cm})$ and mean left ventricular ejection fraction $(65$ (12)\%) did not differ between the four groups. Left ventricular dysfunction (ejection fraction $<50 \%$ ) was present in $9 \%$ of the patients; this proportion tended to be higher in group $3(26 \%)(p=0.11)$.

At the time of ablation 74 patients $(38 \%)$ received class I medication and 96 patients $(49 \%)$ received class III medication. The majority $(\mathrm{n}=136,69 \%)$ also received atrioventricular nodal slowing drugs $(\beta$ blocker, verapamil, or digitalis). Only 38 patients $(19 \%)$ did not take any antiarrhythmic drug. Class I drugs were taken predominantly by patients of group $3(47 \%)$ and $4(57 \%)$ compared with group $1(33 \%)$ and group $2(24 \%)(p=0.02)$. There was no significant difference in the use of the other classes of drugs.

The median (interquartile range) follow up duration for the 196 patients was $2.2(2.4)$ years. Six patients died, none of arrhythmia or procedure related causes. The ablation procedure was successful in $98.5 \%$ - that is, it achieved bidirectional conduction block over the isthmus for at least 30 minutes. In three of 196 patients only unidirectional block was achieved. Two of them remained free of AFL after two and five years of follow up. The third patient developed recurrence after 60 days and, since he also had end stage primary pulmonary hypertension, the procedure was not repeated. Seven patients $(4 \%)$ with procedural bidirectional block developed recurrence between 2-150 months after the initial procedure. Five underwent repeat ablation and two a third procedure to ablate the AFL permanently. Two patients did not have a repeat ablation, since they had only one AFL recurrence.

\section{AF development and its risk factors}

AF developed in 114 patients (58\%). Fourteen patients (7\%) developed permanent $\mathrm{AF}, 12(6 \%)$ persistent $\mathrm{AF}$, and 88 (45\%) paroxysmal AF. In $57 \%$ of patients with postprocedural $\mathrm{AF}$, AF occurred within the first month after the ablation (fig 1A) (median (interquartile range) 24 (175) days). There was a significant difference in AF development between the groups (fig 1B). Group 1 patients (true AFL) had the best prognosis and they had a significantly lower risk for AF in comparison with the other groups. The relative risk ratios to develop AF were 1.9, 3.1, and 2.0 for patients in groups 2, 3, and 4 , respectively, in comparison with patients from group 1 $(\mathrm{p}<0.01$ for each). Forty one per cent of group 1 patients developed AF after one year versus $67 \%, 76 \%$, and $58 \%$, respectively, in groups 2, 3, and 4. After two years these percentages were $43 \%, 74 \%, 91 \%$, and $72 \%$ for patients in groups 1, 2, 3, and 4, respectively. Group 3 patients had a significantly higher chance to develop permanent AF (16\%) or persistent $\mathrm{AF}(16 \%)$ than the other groups (permanent $\mathrm{AF}$ $7 \%$, persistent AF 6\%) (p<0.01).

Univariate and multivariate analyses showed that the presence of preprocedural AF was the strongest predictor of the occurrence of AF after ablation (table 2). Other significant factors were the number of antiarrhythmic drugs used before ablation and a history of cardioversion.

\section{Relation between drug use and AF development}

Table 3 summarises the relation between the use of several types of drugs and AF development. The analysis was based on intention to treat. Patients were classified into a given drug group when the medication was given on or before day 0 and continued until at least day 30 . The main reason for the use of renin-angiotensin pathway blockers was hypertension $(\mathrm{n}=24,52 \%)$ followed by non-ischaemic $(\mathrm{n}=9,20 \%)$ and ischaemic cardiomyopathy $(\mathrm{n}=8,17 \%)$, and valvar heart diseases $(n=5,11 \%)$. Diuretics were used mainly to treat hypertension $(\mathrm{n}=12,44 \%)$, ischaemic $(\mathrm{n}=6,22 \%)$ and non-ischaemic ( $\mathrm{n}=4,15 \%)$ cardiomyopathy, valvar heart disease $(\mathrm{n}=4,15 \%)$, and primary pulmonary hypertension $(\mathrm{n}=1,4 \%)$. A higher proportion of patients taking ACE inhibitors/ARBs were also taking class III drugs and a lower proportion class I drugs. Also among the patients taking diuretics a higher proportion were taking class III drugs (table 4).

The use of ACE inhibitors/ARBs or diuretics before ablation was correlated with a significantly lower rate of AF development, with relative risk ratios of $0.62(p=0.04)$ and $0.34(\mathrm{p}<0.01)$, respectively (table 3 , fig 2$)$. Nevertheless, these patients had a higher proportion of structural heart disease: $100 \% \quad v \quad 37 \% \quad(\mathrm{p}<0.01)$. The combination of ACE inhibitors/ARBs and diuretics was associated with a relative AF risk reduction to 0.22 compared with patients not treated with these medications (only 7\% and $16 \%$ developed AF after one and two years of follow up, respectively). This risk reduction persisted after multivariate analysis. Even after adjusting for age, structural heart disease, preprocedural $\mathrm{AF}$, number of antiarrhythmic drugs used before, a history of cardioversion, and the use of class I or class III medication, there was still a significant benefit 
Table 1 Baseline characteristics of the patients

\begin{tabular}{|c|c|c|c|c|c|c|}
\hline & Overall & $\begin{array}{l}\text { Group } 1 \\
\text { (true AFL) }\end{array}$ & $\begin{array}{l}\text { Group } 2 \\
\text { (AFL>AF) }\end{array}$ & $\begin{array}{l}\text { Group } 3 \\
\text { (AF>AFL) }\end{array}$ & $\begin{array}{l}\text { Group } 4 \\
\text { (class Ic AFL) }\end{array}$ & p Value \\
\hline Number & 196 & 84 & 49 & 19 & 44 & \\
\hline Men/women & $160 / 36$ & $69 / 15$ & $40 / 9$ & $14 / 5$ & $37 / 7$ & NS \\
\hline Age (years) & 57 (12) & $58(12)$ & $58(12)$ & $51(11)$ & $57(10)$ & NS \\
\hline Left atrial diameter $(\mathrm{cm})$ & $4.0(0.6)$ & $4.1(0.8)$ & $3.9(0.5)$ & $4.0(0.5)$ & $4.1(0.6)$ & NS \\
\hline Ejection fraction (\%) & 65 (12) & $65(11)$ & $66(13)$ & $62(11)$ & $65(12)$ & NS \\
\hline Structural heart disease & 101 (51\%) & $49(47 \%)$ & $27(55 \%)$ & $9(47 \%)$ & $20(45 \%)$ & NS \\
\hline Hypertension & $34(17 \%)$ & $13(15 \%)$ & $7(14 \%)$ & $3(16 \%)$ & $11(25 \%)$ & NS \\
\hline Ischaemic & $30(15 \%)$ & $16(19 \%)$ & $8(16 \%)$ & $4(21 \%)$ & $2(5 \%)$ & NS \\
\hline Valvar & $19(9 \%)$ & $11(13 \%)$ & $5(10 \%)$ & $0(0 \%)$ & $3(7 \%)$ & NS \\
\hline Congestive heart failure & $8(4 \%)$ & $2(2 \%)$ & $3(6 \%)$ & $0(0 \%)$ & $3(7 \%)$ & NS \\
\hline Congenital heart disease & $5(3 \%)$ & $2(2 \%)$ & $3(6 \%)$ & $0(0 \%)$ & $0(0 \%)$ & NS \\
\hline Hypertrophic obstructive CMP & $3(2 \%)$ & $1(1 \%)$ & $0(0 \%)$ & $1(5 \%)$ & $1(2 \%)$ & NS \\
\hline Primary pulmonary hypertension & $1(0.5 \%)$ & $1(1 \%)$ & $0(0 \%)$ & $0(0 \%)$ & $0(0 \%)$ & NS \\
\hline Cor pulmonale & $1(0.5 \%)$ & $1(1 \%)$ & $0(0 \%)$ & $0(0 \%)$ & $0(0 \%)$ & NS \\
\hline Duration of symptoms (years) & $4.6(4.8)$ & $3.7(3.7)$ & $6.3(6.2)$ & $4.6(4.8)$ & $4.1(4.5)$ & 0.02 \\
\hline Number of antiarrhythmic drugs & $2.8(1.5)$ & $2.5(1.4)$ & $3.0(1.5)$ & $3.7(2.1)$ & $2.9(2.1)$ & 0.01 \\
\hline History of electrical cardioversion & $108(55 \%)$ & $50(60 \%)$ & $22(45 \%)$ & $9(47 \%)$ & $27(61 \%)$ & NS \\
\hline
\end{tabular}

with ACE inhibitors/ARBs (relative risk 0.55, $\mathrm{p}=0.04$ ), diuretics (relative risk 0.28, $\mathrm{p}<0.01$ ), or their combination (relative risk 0.19, $\mathrm{p}<0.01$ ).

The risk reduction was even more pronounced in the subgroup of patients who had AF before the ablation procedure. The relative risk in this group of patients was reduced with univariate analysis to $0.30(\mathrm{p}<0.01), 0.14(\mathrm{p}<0.01)$, and $0.08(\mathrm{p}=0.01)$ for patients taking ACE inhibitors/ARBs, diuretics, and their combination, respectively (fig 3). Also when we corrected for the abovementioned risk factors, the relative risk reduction remained significant in this subgroup of patients (ACE inhibitors/ARBs 0.25, p < 0.01; diuretics $0.12, \mathrm{p}<0.01$; combination 0.09, $\mathrm{p}=0.02$ ).

The use of other antihypertensive drugs ( $\beta$ blocker, dihydropyridine-type calcium antagonists, moxonidine, clonidine) was not associated with risk reduction in the incidence of AF. Also, atrioventricular nodal slowing ( $\beta$ blocker, verapamil, digitalis) had no effect. On the other hand class I drugs were associated with an increased incidence of $\mathrm{AF}$ (table 3 ) by both univariate and multivariate analyses.

Twenty eight per cent of patients were taking warfarin through follow up $(\mathrm{n}=54)$ and $38 \%$ of the patients continued aspirin $(\mathrm{n}=74)$. No patient developed a stroke periprocedurally but two patients $(1 \%)$ developed a stroke after one and four years. They were both in group 1. Neither of these patients received long term anticoagulation. These two patients received aspirin at a daily dose of 300 and $250 \mathrm{mg}$. The first patient was known to have paroxysmal AF after ablation but, since he had no structural heart disease,

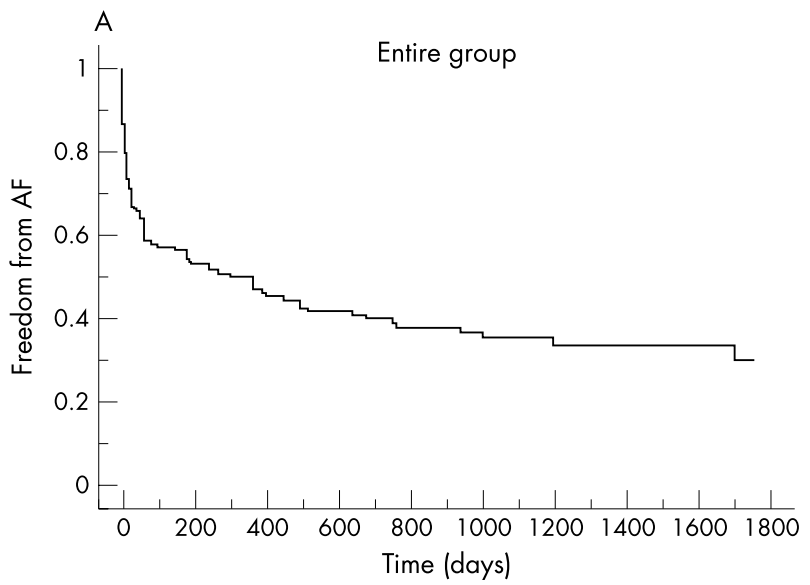

Freedom from $\mathrm{AF}(\%)$

\begin{tabular}{|l|c|c|}
\cline { 2 - 3 } \multicolumn{1}{c|}{} & 1 year & 2 years \\
\hline Entire group & 47 & 40 \\
\hline
\end{tabular}

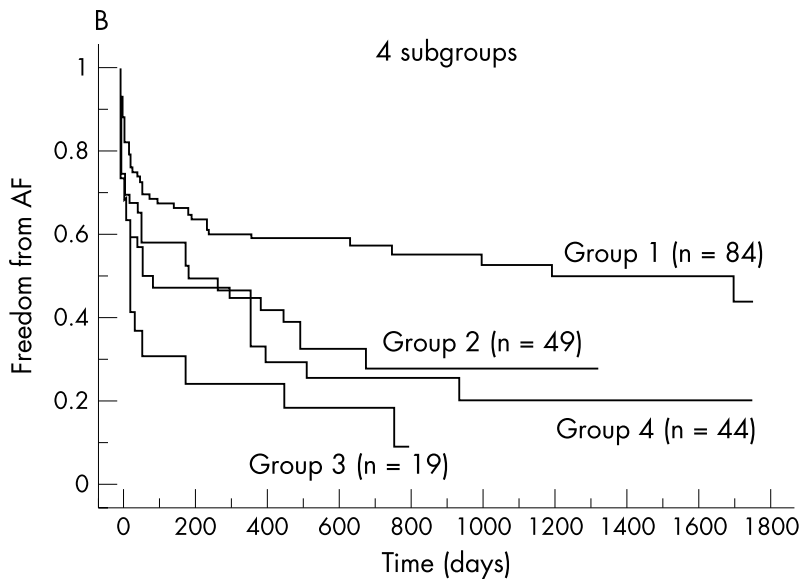

Freedom from $\mathrm{AF}(\%)$

\begin{tabular}{|l|c|c|}
\cline { 2 - 3 } \multicolumn{1}{c|}{} & 1 year & 2 years \\
\hline Group 1 & 59 & 57 \\
\hline Group 2 & 33 & 26 \\
\hline Group 3 & 24 & 9 \\
\hline Group 4 & 42 & 28 \\
\hline
\end{tabular}

Figure 1 (A) Atrial fibrillation (AF) -free survival in the entire study population. (B) AF-free survival in four subgroups: group 1, patients with only atrial flutter (AFL) before the ablation procedure; group 2, predominant AFL but AF occasionally observed; group 3, predominant $A F$ with intermittent organisation into AFL; group 4, class Ic flutter (conversion of AF to AFL caused by treatment with class 1 drugs or amiodarone). Patients from groups 2 , 3 , and 4 each have significantly more $\mathrm{AF}$ than do patients from group 1 (probability values between group 1 and group 2,3 , or 4 are all $\mathrm{p}<0.01$ ). 
Table 2 Factors related to the development of AF after AFL ablation

\begin{tabular}{|c|c|c|c|c|c|c|}
\hline & \multicolumn{3}{|c|}{ Univariate analysis } & \multicolumn{3}{|c|}{ Multivariate analysis } \\
\hline & p Value & RR & $95 \% \mathrm{Cl}$ & p Value & RR & $95 \% \mathrm{Cl}$ \\
\hline Preprocedural AF & $<0.01$ & 2.25 & 1.50 to 3.40 & $<0.01$ & 2.14 & 1.43 to 3.22 \\
\hline Cardioversion & $<0.01$ & 1.72 & 1.19 to 2.49 & $<0.01$ & 1.74 & 1.20 to 2.53 \\
\hline Number of antiarrhythmic drugs & $<0.01$ & 1.19 & 1.05 to 1.34 & $<0.01$ & 1.18 & 1.06 to 1.33 \\
\hline Number of years of atrial flutter & 0.07 & 1.04 & 0.99 to 1.07 & NA & NA & NA \\
\hline Procedural AF & 0.07 & 1.42 & 0.97 to 2.08 & NA & NA & NA \\
\hline Age & 0.11 & 0.99 & 0.98 to 1.01 & NA & NA & NA \\
\hline Structural heart disease & 0.25 & 1.24 & 0.86 to 1.80 & NA & NA & NA \\
\hline Ejection fraction & 0.43 & 0.99 & 0.98 to 1.01 & NA & NA & NA \\
\hline Left atrial diameter & 0.76 & 0.96 & 0.72 to 1.27 & NA & NA & NA \\
\hline
\end{tabular}

hypertension, or diabetes and was 54 years old we decided not give him anticoagulation treatment. The last patient was 67 years old with ischaemic heart disease and hypertension but, since he had no evidence of AF after the procedure, he received only aspirin.

\section{DISCUSSION \\ Main findings}

In this retrospective study concerning the incidence of $\mathrm{AF}$ after ablation for AFL, we found, firstly, that AF often occurs after flutter ablation; secondly, that its incidence is related to pre-ablation AF and its persistence (number of antiarrhythmic drugs used before, a history of cardioversion); thirdly, that blockade of the renin-angiotensin pathway and diuretics are associated with significantly less AF (despite more structural heart disease); and fourthly, that class I drugs have a negative effect on the occurrence of AF.

\section{AF development after AFL ablation}

Both AF and AFL are the expression of the same underlying electrical and structural atrial disease. Most AF develops in the first month after flutter ablation (57\%) but the risk of developing AF later remains substantial. Our recurrence rate is higher than previously reported in the literature $(58 \% v 12-$ $54 \%) .^{2-10}$ This may be attributable to a longer and more intense follow up or to a different proportion of patients with risk factors to develop AF. Our recurrence rate may, however, by itself have been underestimated, since an unknown proportion of patients may have developed asymptomatic AF. ${ }^{19}{ }^{20}$ Only a minority of the patients developed persistent or permanent AF. Patients with a predominance of AF before the procedure (group 3) had a significantly higher risk of developing persistent or permanent AF. Despite the high incidence of post-ablation AF, only two patients developed stroke (after one year and four years). It was judged that they did not require long term anticoagulation (they had no clinical risk profile or were free from AF). This finding may, however, raise the question of whether a more systematic anticoagulation strategy should be adopted after flutter ablation, especially in the group of patients who already had AF before their flutter ablation or who have clinical risk indicators. Only larger, prospective studies can address this question.

Many variables have been identified that are predictive of the development of AF after flutter ablation. ${ }^{2-10}$ We defined pre-ablation $\mathrm{AF}$, the number of antiarrhythmic drugs, and a history of cardioversion as risk factors; others have pointed also to structural heart disease, inducibility of AF during the procedure, and left atrial diameter and ejection fraction as prognostic factors. ${ }^{245810}$ What all of these factors have in common is their relation to the degree of underlying electrical and structural damage to the atria.

\section{Renin-angiotensin pathway and $\mathrm{AF}$}

The use of renin-angiotensin blockers (ACE inhibitors/ARBs) and diuretics was associated with less AF development, although these drugs were taken significantly more often by patients with underlying structural heart disease. Although there was a higher use of class III drugs among patients taking ACE inhibitors/ARBs or diuretics, the beneficial effects of ACE inhibitors/ARBs and diuretics remained significant after correcting for class III drug use. In various animal models of AF, the role of angiotensin II in the development of the atrial substrate of AF and its prevention by ACE inhibitors or ARBs has been indicated..$^{12}{ }^{13}$ Also, ACE inhibitors led to a reduction in AF development after myocardial infarction and in a heart failure population, and ARBs have been shown to reduce the recurrence rate after cardioversion. $^{14-16}$ Inhibition of angiotensin II can be preventive through several mechanisms: lowering of arterial blood pressure, lowering of atrial pressure and stretch, and direct inhibition of cardiac fibrogenesis. ${ }^{13}$ Since other antihypertensive drugs do not have this preventive effect, probably the direct inhibition of cardiac fibrogenesis provides the most important explanation. By activating the mitogen activated protein kinases, angiotensin II induces the proliferation of

Table 3 Post-ablation drug use and relation with AF development

\begin{tabular}{|c|c|c|c|c|c|}
\hline & \multirow{2}{*}{$\begin{array}{l}\text { Number of } \\
\text { patients }\end{array}$} & \multicolumn{2}{|l|}{ Univariate analysis } & \multicolumn{2}{|l|}{ Multivariate analysis } \\
\hline & & $\operatorname{RR}(95 \% \mathrm{Cl})$ & $\mathrm{p}$ Value & $\operatorname{RR}(95 \% \mathrm{Cl})$ & $\mathrm{p}$ Value \\
\hline Class I drugs & $56(29 \%)$ & 1.96 (1.33 to 2.87 ) & $<0.01$ & 1.66 (1.06 to 2.60$)$ & 0.03 \\
\hline Class III drugs & $74(38 \%)$ & 0.86 (0.58 to 1.26$)$ & 0.43 & $0.76(0.50$ to 1.19$)$ & 0.23 \\
\hline $\mathrm{AV}$ node slowing drugs & $85(43 \%)$ & $1.22(0.85$ to 1.78$)$ & 0.29 & $0.97(0.66$ to 1.44$)$ & 0.89 \\
\hline ACE inhibitors/ARB & $46(23 \%)$ & $0.62(0.37$ to 0.99$)$ & 0.04 & $0.55(0.31$ to 0.97$)$ & 0.04 \\
\hline Diuretics & $27(14 \%)$ & $0.34(0.16$ to 0.70$)$ & $<0.01$ & $0.28(0.13$ to 0.63$)$ & $<0.01$ \\
\hline Other antihypertensive drugs & $85(43 \%)$ & $1.01(0.73$ to 1.64 ) & 0.67 & 1.00 (0.70 to 1.58$)$ & 0.83 \\
\hline
\end{tabular}

Intention to treat analysis. A patient was classified into a given drug group when the medication was given on day 0 and continued until at least day 30 Atrioventricular (AV) node slowing drugs: $\beta$ blockers, verapamil, digitalis. Other antihypertensive drugs: $\beta$ blocker, dihydropyridine-type calcium antagonists, moxonidine, clonidine.

$\mathrm{ACE}$, angiotensin converting enzyme; ARB: angiotensin II receptor blockers. 
Table 4 Use of ACE inhibitors and diuretics

\begin{tabular}{|c|c|c|c|c|c|c|}
\hline & \multicolumn{3}{|c|}{ ACE inhibitors } & \multicolumn{3}{|l|}{ Diuretics } \\
\hline & Yes $(n=46)$ & No $(n=150)$ & p Value & Yes $(n=27)$ & No $(n=169)$ & p Value \\
\hline Class I & $6(13 \%)$ & $50(33 \%)$ & $<0.01$ & $4(15 \%)$ & $52(31 \%)$ & 0.08 \\
\hline Class III & $28(61 \%)$ & $46(31 \%)$ & $<0.01$ & $16(59 \%)$ & $58(34 \%)$ & 0.01 \\
\hline$\beta$ Blocker & $10(21 \%)$ & $38(25 \%)$ & 0.62 & $10(37 \%)$ & $38(22 \%)$ & 0.10 \\
\hline Calcium antagonist & $5(11 \%)$ & $33(22 \%)$ & 0.09 & $5(18 \%)$ & 33 (19\%) & 0.90 \\
\hline Digitalis & $7(15 \%)$ & $15(10 \%)$ & 0.32 & $6(22 \%)$ & $16(9 \%)$ & 0.05 \\
\hline
\end{tabular}

fibroblasts, as well as extracellular matrix protein accumulation. ${ }^{11}$ In a dog model of AF, Li and colleagues ${ }^{13}$ proved that ACE inhibitors prevented atrial fibrosis; this was associated with decreased phosphorylation of extracellular signal kinases, which are members of the mitogen activated protein kinase family.

The effect of ACE inhibition on the prevention of AF development was already seen within one month after ablation (fig 2, fig 3). This can probably be explained by the fact that the majority of patients $(80 \%)$ already took ACE inhibitors/ARBs before ablation, allowing foregoing structural remodelling. Also, other studies of the effect of ACE inhibitors/ARBs on AF development showed rapidly diverging Kaplan-Meier curves. ${ }^{15}{ }^{16}$ These data may suggest that besides structural remodelling ACE inhibition also prevents AF by electrical effects. On the other hand, experimentation can observe an electrical effect only in the acute setting, not in the longer term, ${ }^{21-23}$ indicating that structural remodelling certainly is determinant in the longer term.

The effect of diuretics in the prevention of AF is less clear. It can be attributed to lowering blood pressure and diminishing atrial preload, both leading to decreased stretch. On the other hand, diuretics activate the renin-angiotensin-aldosterone system. ${ }^{24}$ This may explain why the combination of diuretics and renin-angiotensin pathway blockers was associated with the most pronounced relative risk reduction for the development of AF (0.20). Our findings may provide impetus for prospective evaluation of this combined intervention.

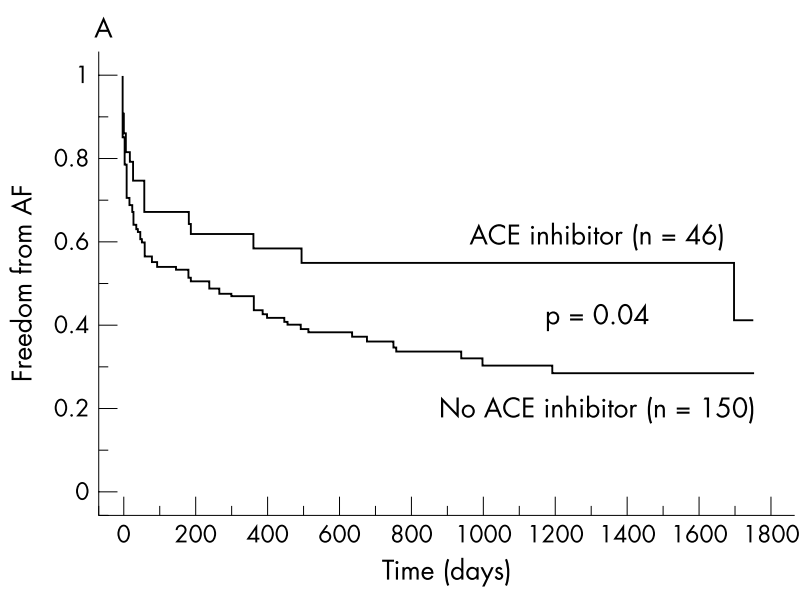

Freedom from AF (\%)

\begin{tabular}{|r|c|c|}
\cline { 2 - 3 } \multicolumn{1}{c|}{} & 1 year & 2 years \\
\hline ACE inhibition & 59 & 55 \\
\hline No ACE inhibition & 44 & 36 \\
\hline
\end{tabular}

\section{Class I drugs and AF}

The positive association between class I drugs and AF development may be anticipated because of inclusion bias (that is, patients with a higher risk profile would be prescribed the drugs). However, the association remained significant after multivariate analysis (table 3). The effect of class I drugs after AFL ablation has never been studied prospectively. It is possible that there is heterogenic slowing of conduction velocity in these atria, which is proarrhythmogenic. ${ }^{25}$ Further study is required to evaluate the treatment potential of class I drugs in (subsets of) patients with AFL.

\section{Limitations}

The frequency of postprocedural and preprocedural AF may have been underestimated because of asymptomatic and brief paroxysmal episodes of AF. Studies have indicated that systematic long term ECG recordings show much higher rates of AF recurrence than does clinical follow up with intermittent ECG or Holter recordings. ${ }^{19} 20$ The fact that our study population is comparable with those of other studies ${ }^{2-10}$ and that the AF rate we found is higher than reported previously suggest that our follow up may at least have been as intensive. On the other hand some symptoms we regarded as AF may have been caused by other arrhythmias. It is unknown to what extent these "misclassifications", inherent to all studies of AF during clinical follow up, may have affected our conclusions.

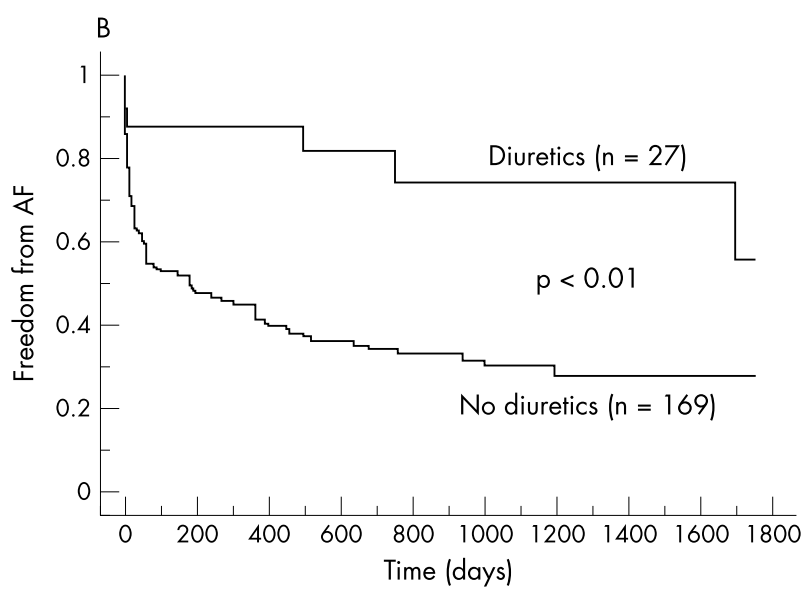

Freedom from AF $(\%)$

\begin{tabular}{|r|c|c|}
\cline { 2 - 3 } \multicolumn{1}{c|}{} & 1 year & 2 years \\
\hline Diuretics & 88 & 74 \\
\hline No diuretics & 41 & 34 \\
\hline
\end{tabular}

Figure 2 (A) AF-free survival among patients taking angiotensin converting enzyme (ACE) inhibitors post-ablation versus those not taking ACE inhibitors. (B) AF-free survival among patients taking diuretics post-ablation versus those not taking diuretics. 


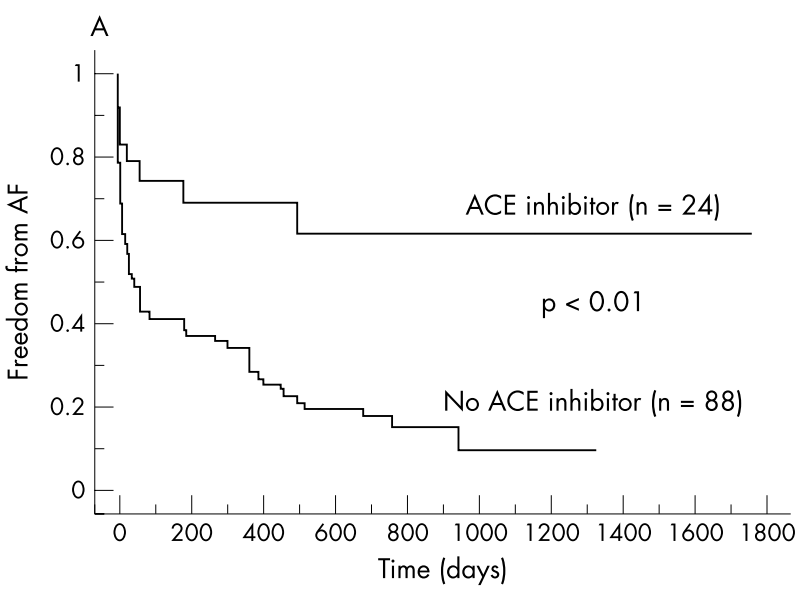

Freedom from AF $(\%)$

\begin{tabular}{|r|c|c|}
\cline { 2 - 3 } \multicolumn{1}{c|}{} & 1 year & 2 years \\
\hline ACE inhibition & 69 & 62 \\
\hline No ACE inhibition & 39 & 18 \\
\hline
\end{tabular}

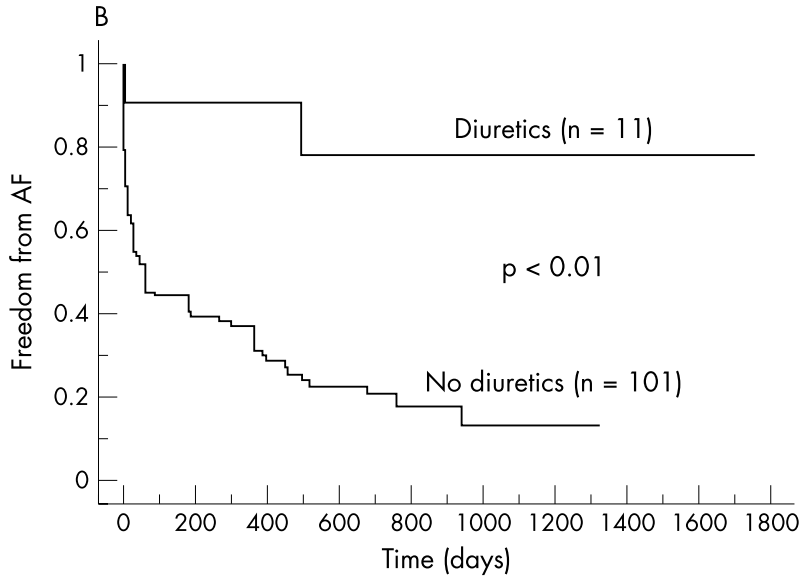

Freedom from AF $(\%)$

\begin{tabular}{|r|c|c|}
\cline { 2 - 3 } \multicolumn{1}{c|}{} & 1 year & 2 years \\
\hline Diuretics & 91 & 78 \\
\hline No diuretics & 31 & 20 \\
\hline
\end{tabular}

Figure 3 (A) AF-free survival among patients taking ACE inhibitors post-ablation versus those not taking ACE inhibitors in the subgroup of patients with AF before the procedure. (B) AF-free survival among patients taking diuretics post-ablation versus those not taking diuretics in the subgroup of patients with $\mathrm{AF}$ before the procedure.

Moreover, since this study was retrospective in nature, only statements about associations can be made, without necessarily implicating causative mechanisms.

\section{Conclusions}

More than half of the patients undergoing ablation for AFL later developed AF. Retrospective analysis showed that factors pointing to more advanced electrical and structural remodelling are associated with an increased risk (preprocedural AF, history of cardioversion, number of antiarrhythmic drugs used before the procedure). On the other hand the use of ACE inhibitors/ARBs and diuretics was associated with less $\mathrm{AF}$, indicating a preventive effect.

\section{Authors' affiliations}

W Anné, Institute for the Promotion of Innovation by Science and Technology, Flanders, Belgium

R Willems, N Van der Merwe, F Van de Werf, H Ector, Department of Cardiology, University Hospital Gasthuisberg, University of Leuven, Leuven, Belgium

H Heidbüchel, Fund for Scientific Research, Flanders, Belgium

\section{REFERENCES}

1 Natale A, Newby KH, Pisano E, et al. Prospective randomized comparison of anti-arrhythmic therapy versus first-line radiofrequency ablation in patients with AFL. J Am Coll Cardiol 2000;35:1898-904

2 Philippon F, Plumb VJ, Epstein AE, et al. The risk of AF following radiofrequency catheter ablation of AFL. Circulation 1995;92:430-5.

3 Saxon LA, Kalman JM, Olgin JE, et al. Results of radiofrequency catheter ablation for AFL. Am J Cardiol 1996:77:1014-6.

4 Frey B, Kreiner G, Binder T, et al. Relation between left atrial size and secondary atrial arrhythmias after successful catheter ablation of common AFL. Pacing Clin Electrophysiol 1997;20:2936-42.

5 Tai CT, Chen SA, Chiang CE, et al. Long-term outcome of radiofrequency catheter ablation for typical AFL: risk prediction of recurrent arrhythmias. J Cardiovasc Electrophysiol 1998;9:115-21.

6 Reithmann C, Hoffmann E, Spitzlberger G, et al. Catheter ablation of AFL due to amiodarone therapy for paroxysmal AF. Eur Heart J 2000;21:565-72.

7 Nabar A, Rodriguez LM, Timmermans C, et al. Effect of right atrial isthmus ablation on the occurrence of AF: observations in four patient groups having type I AFL with or without associated AF. Circulation 1999;99:1441-5.

8 Paydak H, Kall JG, Burke MC, et al. AF after radiofrequency ablation of type I AFL: time to onset, determinants, and clinical course. Circulation 1998;98:315-22.
9 Anselme F, Saoudi N, Poty $\mathrm{H}$, et al. Radiofrequency catheter ablation of common AFL: significance of palpitations and quality-of-life evaluation in patients with proven isthmus block. Circulation 1999;99:534-40.

10 Da Costa A, Romeyer C, Mourot S, et al. Factors associated with early AF after ablation of common AFL: a single centre prospective study. Eur Heart $J$ 2002;23:498-506.

11 Goette A, Staack T, Rocken C, et al. Increased expression of extracellular signal-regulated kinase and angiotensin-converting enzyme in human atria during atrial fibrillation. J Am Coll Cardiol 2000;35:1669-77.

12 Willems R, Sipido KR, Holemans P, et al. Different patterns of angiotensin II and atrial natriuretic peptide secretion in a sheep model of AF. J Cardiovasc Electrophysiol 2001;12:1387-92.

13 Li D, Shinagawa K, Pang L, et al. Effects of angiotensin-converting enzyme inhibition on the development of the AF substrate in dogs with ventricular tachypacing-induced congestive heart failure. Circulation 2001; 104:2608-14.

14 Pedersen OD, Bagger $H$, Kober $L$, et al. Trandolapril reduces the incidence of AF after acute myocardial infarction in patients with left ventricular dysfunction. Circulation 1999; 100:376-80.

15 Vermes E, Tardif JC, Martial GB, et al. Enalapril decreases the incidence of atrial fibrillation in patients with left ventricular dysfunction. Circulation 2003; 107:2926-31

16 Madrid AH, Bueno MG, Rebollo JM, et al. Use of irbesartan to maintain sinus rhythm in patients with long-lasting persistent AF: a prospective and randomized study. Circulation 2002;106:331-6.

17 Schumacher B, Jung W, Lewalter T, et al. Radiofrequency ablation of atrial flutter due to administration of class IC anti-arrhythmic drugs for atrial fibrillation. Am J Cardiol 1999:34:710-3.

18 Heidbuchel $\mathrm{H}$, Willems $\mathrm{R}$, van Rensburg $\mathrm{H}$, et al. Right atrial angiographic evaluation of the posterior isthmus: relevance for ablation of typical atrial flutter. Circulation 2000;101:2178-84.

19 Defaye $\mathbf{P}$, Dournaux F, Mouton E. Prevalence of supraventricular arrhythmias from the automated analysis of data stored in the DDD pacemakers of 617 patients: the AIDA study: the AIDA multicenter study group. Automatic interpretation for diagnosis assistance. Pacing Clin Electrophysiol 1998;21:250-5.

20 Glotzer TV, Hellkamp AS, Zimmerman J, et al. Atrial high rate episodes detected by pacemaker diagnostics predict death and stroke: report of the atrial diagnostics ancillary study of the mode selection trial (MOST). Circulation 2003;107:1614-9.

21 Nakashima H, Kumagai K, Urata $\mathrm{H}$, et al. Angiotensin II antagonist prevents electrical remodeling in atrial fibrillation. Circulation 2000;101:1436-8.

22 Kumagai K, Nakashima $\mathrm{H}$, Urata $\mathrm{H}$, et al. Effects of angiotensin II type 1 receptor antagonist on electrical and structural remodeling in atrial fibrillation. J Am Coll Cardiol 2003;41:2197-204.

23 Shinagawa K, Mitamura $\mathrm{H}$, Ogawa S, et al. Effects of inhibiting $\mathrm{Na}(+) / \mathrm{H}(+)-$ exchange or angiotensin converting enzyme on atrial tachycardia-induced remodelling. Cardiovasc Res 2002;54:438-46.

24 Ellison DH. Diuretic therapy and resistance in congestive heart failure. Cardiology 2001;96:132-43.

25 Li D, Fareh S, Leung TK, et al. Promotion of atrial fibrillation by heart failure in dogs: atrial remodeling of a different sort. Circulation 1999;100:87-95. 